\title{
TRANSFERÊNCIA DE ENERGIA ENTRE CORANTES CATIÔNICOS EM SISTEMAS HOMOGÊNEOS
}

Hueder Paulo Moisés de Oliveira, Amilcar Machulek Junior, Alexandre de Oliveira Legendre e Marcelo Henrique Gehlen* Instituto de Química de São Carlos, Universidade de São Paulo, Av. Trabalhador Sãocarlense, 400, 13566-590 São Carlos - SP

Recebido em 2/8/02; aceito em 12/12/02

\begin{abstract}
ENERGY TRANSFER BETWEEN CATIONIC DYES IN HOMOGENEOUS SYSTEMS. In this work, the energy transfer by dipole-dipole interaction between cationic dyes in $n$-alcohols (methanol, ethanol, 1-propanol and 1-butanol) is studied by time resolved and steady state fluorescence measurements. The critical radii of energy transfer were determined by three independent methods; the spectral overlap, fluorescence decay profiles, and relative intensity measurements. In all solvents, $R_{0}$ values of the dye pairs obtained from spectral overlap were between 40 to $90 \AA$. Steady state and time resolved fluorescence measurements resulted in values of $R_{0}$ in the range of $50-80 \AA$, with good correlation of values.
\end{abstract}

Keywords: dyes; fluorescence; energy transfer.

\section{INTRODUÇÃO}

O estudo de transferência de energia entre corantes tem importância em vários meios como na conversão de energia solar e em lasers ${ }^{1-3}$. A transferência de energia é uma etapa primária em processos de interesse biológico (como a fotossíntese), bem como em sistemas de interesse industrial (fotopolimerização) ${ }^{4}$. Transferência de energia pelo mecanismo de Förster permite determinar o número de agregação de micelas em sistemas de detergentes ${ }^{1-7}$, bem como determinar a conformação de polímeros em solução. A dependência da transferência de energia com a distância permite utilizá-la como uma ferramenta para conjecturar sobre a distribuição espacial de moléculas solubilizadas em micelas e polímeros ${ }^{8-12}$. O raio crítico de Förster para a maioria dos sistemas estudados está na faixa de 30-90 $\AA^{8}$.

O processo de transferência de energia pelo mecanismo dipolar pode ser descrito segundo as seguintes etapas:

$D+h \vee \rightarrow D^{*}$

$D^{*}+A \stackrel{k_{R}}{\longrightarrow} D+A^{*}$

A interação dipolo-dipolo é proporcional ao momento de transição $\vec{M}$ e inversamente proporcional à distância ao cubo. Assim, a transferência de energia é um processo dinâmico no qual a velocidade de transferência depende da distância entre o doador e o receptor. A constante de velocidade de transferência é dada por ${ }^{1,2,8,11,12}$ :

$k(r)=\frac{3}{2} \kappa^{2}\left(\frac{R_{o}}{r}\right)^{6} \tau_{o}^{-1}$

onde: $\mathrm{R}_{\mathrm{o}}$ é o raio crítico de Förster, $\tau_{\mathrm{o}}$ é o tempo de vida de fluorescência na ausência de supressor, $r$ é a distância entre o doador e o receptor e $\kappa$ é o fator de orientação entre os dipolos do doador excitado e do receptor ${ }^{13,14}$.

No regime dinâmico de rápida rotação $\kappa^{2}$ converge em média para $2 / 3$. O raio de Förster $\left(R_{0}\right)$ corresponde à distância na qual a probabilidade de transferência é igual à probabilidade de decaimento. A equação proposta por Förster ${ }^{15}$ para se calcular o valor de $R_{0}$ é dada por:

*e-mail: marcelog@iqsc.usp.br
$R_{o}^{6}=\frac{9000(\ln 10) \phi_{D} \kappa^{2}}{128 \pi^{5} N_{a} n^{4}} \int \frac{f(\bar{v})}{\bar{v}^{3}}\left(\frac{\varepsilon}{\bar{v}}\right) d \bar{v}$

Onde o valor do integrando é chamado de integral de recobrimento espectral. Os valores de $\mathrm{R}_{\mathrm{o}}$ também podem ser obtidos a partir de experimentos de fluorescência resolvida no tempo e de medidas fotoestacionárias. $\mathrm{O}$ valor de $\mathrm{R}_{0}$ pela técnica de fluorescência resolvida no tempo é calculado a partir da seguinte equação:

$f(t)=\exp \left[\frac{-t}{\tau_{0}}-2 \Gamma\left(\frac{t}{\tau_{0}}\right)^{\frac{1}{2}}\right]$

Na qual:

$2 \Gamma=\frac{4}{3} \pi^{\frac{3}{2}} n_{a} R_{0}^{3}$

onde $n_{a}$ é a densidade de moléculas de receptor (moléculas $/ \mathrm{cm}^{3}$ ).

De acordo com a teoria de Förster ${ }^{14,15}$, a eficiência da transferência de energia em fase homogênea é dada por:

$E=1-\frac{I_{o}}{I}$

No caso de medidas fotoestacionárias, o valor de $\mathrm{R}_{0}$ é dado por:

$R_{o}=\left(\frac{3000}{11,14 C_{0} N}\right)^{\frac{1}{3}}$

$\mathrm{O}$ valor de $\mathrm{C}_{0}$ (concentração crítica de receptor) é obtido da seguinte equação para a eficiência:

$E=\pi^{\frac{1}{2}}\left(\frac{C}{C_{o}}\right) \exp \left(\frac{C}{C_{o}}\right)^{2}\left[1-\operatorname{erf}\left(\frac{C}{C_{o}}\right)\right]$

onde C é a concentração de supressor (receptor) e erf é a função erro, dada por:

$\operatorname{erf}(m)=\frac{2}{\sqrt{\pi}} \int_{0}^{m} \exp \left(-x^{2}\right) d x$ 


\section{PARTE EXPERIMENTAL}

Os corantes utilizados foram 9-aminoacridina (Aldrich), proflavina (Aldrich), laranja de acridina (Aldrich), azul do Nilo (Aldrich), violeta de cresila (Aldrich), cristal violeta (Aldrich), azul de toluidina (Merck) e safranina (Aldrich). Todos os corantes foram purificados por recristalização em metanol. As medidas foram feitas a $25^{\circ} \mathrm{C}$ e em concentração de $10^{-5} \mathrm{M}$ nos seguintes solventes grau HPLC: metanol, etanol, propanol-1 e butanol-1. Os espectros de absorção foram obtidos em um espectrofotômetro Hitachi U-2000, e os espectros de emissão corrigidos em um espectrofluorímetro Hitachi F-3500. As amostras foram condicionadas em cubetas de quartzo (1 x $1 \mathrm{~cm}$ ). Os valores de rendimento quântico de fluorescência foram determinados por comparação com padrões conhecidos de fluorescência ${ }^{16}$. As concentrações dos corantes foram mantidas abaixo da concentração de auto-agregação dos mesmos, a fim de impedir a interferência de quaisquer outros processos nas análises dos dados obtidos. Os decaimentos de fluorescência dos corantes foram medidos pela técnica de contagem de fótons resolvida no tempo, usando um espectrômetro CD-900 Edinburgh equipado com polarizadores e uma fotomultiplicadora Hamamatsu R955 resfriada a $-25{ }^{\circ} \mathrm{C}$ por um sistema Peltier. Os pulsos de excitação foram gerados a partir de um sistema de lasers Mira $900 \mathrm{Ti}$ - Safira bombeado em $532 \mathrm{~nm}$ por um Verdi $5 \mathrm{~W}$ da Coherent. A freqüência de pulsos de $76 \mathrm{MHz}$ foi reduzida a menos de $1 \mathrm{MHz}$ usando-se um redutor optoeletrônico de pulsos da Conoptics modelos MD 25D/305/350-160. A excitação em $400 \mathrm{~nm}$ foi obtida pela incidência do pulso de laser sobre um gerador de segundo harmônico (construído com um cristal de BBO $1 \mathrm{~mm}$ UTO 8204 Casix) e a temporização do pulso de excitação foi monitorada por um fotodiodo rápido (modelo 818 BB21 da Newport). Os decaimentos dos corantes que absorvem na região de $600 \mathrm{~nm}$ foram medidos no mesmo equipamento, mas com o pulso de excitação gerado pelo diodo laser de 100 ps em 633 nm (Hamamatsu modelo PLP 01). Os decaimentos de fluorescência foram analisados pela convolução da função de resposta do instrumento com os modelos exponencial e cinética de supressão de Förster presentes no "software" da Edinburgh Instruments. A resposta instrumental, definida pela largura da altura média da resposta temporal do detector, foi de aproximadamente $700 \mathrm{ps}$. Os decaimentos foram obtidos com incremento de tempo de $25-50$ ps por canal, com 1 Kbyte de pontos por decaimento.

\section{RESULTADOS E DISCUSSÃO}

\section{Propriedades fotofísicas dos corantes}

As estruturas moleculares dos corantes podem ser vistas na Figura 1.

Com base nos valores da razão $\mathrm{I}_{\mathrm{I}} \mathrm{I}_{\mathrm{III}}$ do pireno, observou-se que a polaridade dos solventes usados diminui com o aumento da cadeia carbônica alifática dos álcoois (1,32, 1,15, 1,04 e 0,99 para metanol, etanol, propanol-1 e butanol-1, respectivamente). Esta diminuição dos valores influi nos deslocamentos dos espectros de absorção e emissão dos corantes, contribuindo para um maior ou menor valor de rendimento quântico de fluorescência (Tabela 1). A dependência dos valores obtidos para o tempo de vida em função da viscosidade e constante dielétrica do solvente indica que o processo de desativação do estado excitado dos corantes torna-se mais lento em meios mais viscosos. Este efeito está relacionado com a inibição parcial ou a maior fricção na rotação de grupos substituintes como $-\mathrm{CH}_{3},-\mathrm{NH}_{2}$, $-\mathrm{N}\left(\mathrm{CH}_{3}\right)_{2}$ e $-\mathrm{N}\left(\mathrm{CH}_{2} \mathrm{CH}_{3}\right)_{2}$ dos corantes. Os valores encontrados para os tempos de vida estão de acordo com dados reportados na literatu$\mathrm{ra}^{17-19}$. Um outro fato a ser considerado é que sondas que apresentam

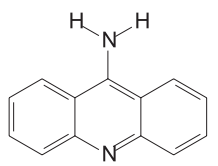

9-Aminoacridina (9AA)
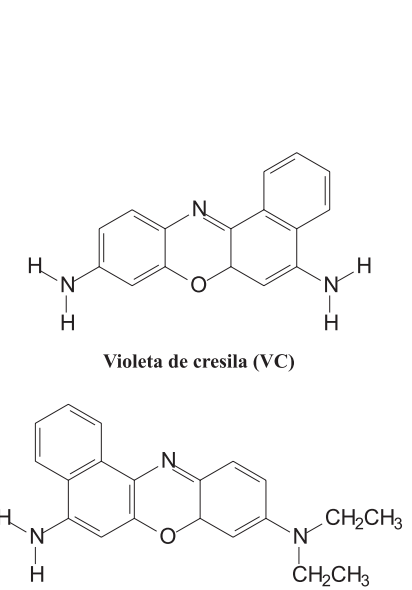

Azul do Nilo (AN)

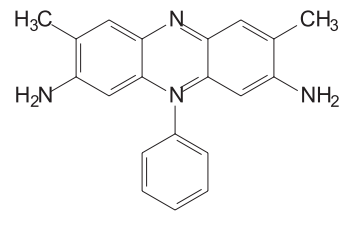

Safranina (SAF)

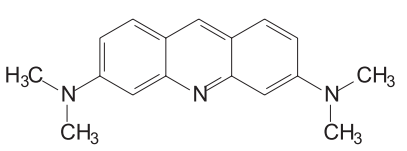

Laranja de acridina (LA)
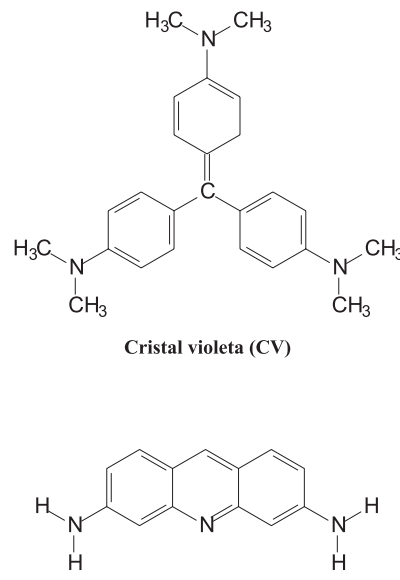

Proflavina (PROF)

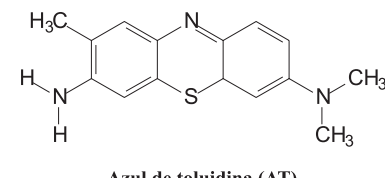

Azul de toluidina (AT)
Figura 1. Fórmulas estruturais dos corantes utilizados

grupamentos $\mathrm{NH}_{2}$ na estrutura, como o azul do Nilo, podem apresentar processos de transferência de carga intramolecular, acarretando também mudanças nos valores de tempo de vida ${ }^{17-21}$. Os valores das constantes de velocidade de desativação radiativa $\left(\mathrm{k}_{\mathrm{r}}\right)$ e nãoradiativa $\left(\mathrm{k}_{\mathrm{nr}}\right)$ ajudam a confirmar que as mudanças observadas nas propriedades espectrais dos corantes são devidas ao processo de transferência de carga intramolecular, como pode ser visto na Tabela 2.

Os valores dos comprimentos de onda máximo de absorção $\left(\lambda_{\text {abs }}\right)$ e emissão $\left(\lambda_{\text {em }}\right)$, e o deslocamento espectral ou de Stokes $(\Delta \bar{v})$ dos corantes dependem da polaridade do solvente, como pode ser visto nos dados apresentados na Tabela 3. Os espectros de absorção e de emissão dos corantes são deslocados pelo efeito de solvente. Os deslocamentos observados para os diversos corantes utilizados estão de acordo com dados presentes na literatura ${ }^{17-21}$.

Há uma dependência linear do deslocamento de Stokes com o parâmetro de polaridade dos solventes $(\Delta f)$, seguindo a equação de Lippert-Mataga (Equação 11) $)^{14,18-20}$.

$\Delta \bar{v}=\frac{2 \Delta \mu^{2}}{c h a^{3}} \Delta f$

Na equação acima, $\Delta \mu^{2}$ é a diferença entre os quadrados dos momentos de dipolo da molécula no estado fundamental e excitado, $a$ é o raio de Onsager da cavidade formada ao redor da molécula, $c$ é a velocidade da luz no vácuo, e $h$ é a constante de Planck. A inclinação da reta depende da diferença do momento de dipolo da molécula antes e depois da excitação, como pode ser visto na Figura 2. A inclinação das retas apresentadas na Figura 2 mostra que $\Delta \mu^{2} \geq 0$ para os sistemas. Este fato indica que o momento de dipolo da molécula no estado excitado é um pouco maior que no estado fundamental. 
Tabela 1. Valores de tempo de vida $(\tau)$ e rendimento quântico de fluorescência $(\phi)$ para os corantes em diferentes álcoois, $\mathrm{T}=298 \mathrm{~K}$

\begin{tabular}{lcccc}
\hline Corante & \multicolumn{3}{c}{$\tau(\mathrm{ns}) ; \phi$} \\
\cline { 2 - 5 } & $\mathrm{MeOH}$ & $\mathrm{EtOH}$ & $\mathrm{PrOH}$ & 3,$02 ; 0,18$ \\
\hline Safranina & 2,$59 ; 0,12$ & 2,$93 ; 0,18$ & 3,$02 ; 0,18$ & 4,$05 ; 0,15$ \\
Laranja de acridina & 2,$95 ; 0,20$ & 3,$38 ; 0,20$ & 3,$65 ; 0,19$ & 11,$82 ; 0,45$ \\
9-Aminoacridina & 13,$89 ; 0,40$ & 13,$10 ; 0,43$ & 12,$57 ; 0,44$ & 4,$52 ; 0,40$ \\
Proflavina & 4,$41 ; 0,42$ & 4,$31 ; 0,42$ & 4,$27 ; 0,41$ & 2,$09 ; 0,16$ \\
Azul do Nilo & 1,$21 ; 0,13$ & 1,$54 ; 0,18$ & 1,$86 ; 0,18$ & 0,$10 ; 5.10-3$ \\
Cristal violeta & 0,$45 ; 4.10^{-3}$ & 0,$28 ; 9.10^{-4}$ & 0,$31 ; 6.10^{-4}$ & 3,$39 ; 0,22$ \\
Violeta de cresila & 3,$31 ; 0,30$ & 3,$36 ; 0,30$ & 3,$40 ; 0,25$ & 6,$00 ; 0,11$ \\
Azul de toluidina & 4,$65 ; 0,09$ & 5,$59 ; 0,10$ & & 6,12 \\
\hline
\end{tabular}

Tabela 2. Valores de constantes de velocidade de desativação radiativa $\left(\mathrm{k}_{\mathrm{r}}\right)$ e não-radiativa $\left(\mathrm{k}_{\mathrm{nr}}\right)$ para os corantes utilizados em diferentes álcoois, T = $298 \mathrm{~K}$

\begin{tabular}{|c|c|c|c|c|c|c|c|c|}
\hline \multirow{3}{*}{$\begin{array}{l}\text { Corante } \\
\text { Safranina }\end{array}$} & \multicolumn{8}{|c|}{$\mathrm{k}_{\mathrm{r}} \mathrm{e} \mathrm{k}_{\mathrm{nr}}, 10^{7} \mathrm{~s}^{-1}$} \\
\hline & \multicolumn{2}{|c|}{$\mathrm{MeOH}$} & \multicolumn{2}{|c|}{$\mathrm{EtOH}$} & \multicolumn{2}{|c|}{$\mathrm{PrOH}$} & \multicolumn{2}{|c|}{$\mathrm{BuOH}$} \\
\hline & 4,63 & 33,98 & 6,14 & 27,99 & 5,96 & 27,15 & 5,96 & 27,15 \\
\hline Laranja de acridina & 6,78 & 27,12 & 5,92 & 23,67 & 5,21 & 22,19 & 3,70 & 20,99 \\
\hline 9-Aminoacridina & 2,88 & 4,32 & 3,28 & 4,35 & 3,50 & 4,46 & 3,81 & 4,65 \\
\hline Proflavina & 9,52 & 13,15 & 9,75 & 13,46 & 9,60 & 13,82 & 8,85 & 13,27 \\
\hline Azul do Nilo & 10,74 & 71,90 & 11,69 & 53,25 & 9,68 & 44,09 & 7,66 & 40,19 \\
\hline Cristal violeta & 0,89 & 221,33 & 0,32 & 356,82 & 0,19 & 322,39 & 5,00 & 995,00 \\
\hline Violeta de cresila & 9,06 & 21,15 & 8,93 & 20,83 & 7,35 & 22,06 & 6,49 & 23,01 \\
\hline Azul de toluidina & 1,94 & 19,57 & 1,79 & 16,10 & 1,83 & 14,83 & 1,76 & 12,92 \\
\hline
\end{tabular}

Tabela 3. Valores de comprimentos de onda máximo de absorção $\left(\lambda_{\mathrm{abs}}\right)$ e emissão $\left(\lambda_{\mathrm{em}}\right)$ em nm, e deslocamento de $\operatorname{Stokes}(\Delta \bar{v})$ para os corantes utilizados, $\mathrm{T}=298 \mathrm{~K}$

\begin{tabular}{|c|c|c|c|c|}
\hline Solvente & $\mathrm{MeOH}$ & $\mathrm{EtOH}$ & $\mathrm{PrOH}$ & $\mathrm{BuOH}$ \\
\hline \multirow[t]{2}{*}{ Safranina } & $528 / 563$ & $533 / 564$ & $536 / 562$ & $537 / 564$ \\
\hline & $1177 \mathrm{~cm}^{-1}$ & $1031 \mathrm{~cm}^{-1}$ & $863 \mathrm{~cm}^{-1}$ & $892 \mathrm{~cm}^{-1}$ \\
\hline \multirow[t]{2}{*}{ Laranja de acridina } & $489 / 519$ & $489 / 517$ & $489 / 517$ & $489 / 517$ \\
\hline & $1182 \mathrm{~cm}^{-1}$ & $1108 \mathrm{~cm}^{-1}$ & $1108 \mathrm{~cm}^{-1}$ & $1108 \mathrm{~cm}^{-1}$ \\
\hline \multirow[t]{2}{*}{ 9-Aminoacridina } & $400 / 454$ & $401 / 454$ & $401 / 454$ & $402 / 454$ \\
\hline & $2973 \mathrm{~cm}^{-1}$ & $2911 \mathrm{~cm}^{-1}$ & $2911 \mathrm{~cm}^{-1}$ & $2849 \mathrm{~cm}^{-1}$ \\
\hline \multirow[t]{2}{*}{ Proflavina } & $456 / 492$ & $459 / 492$ & $459 / 491$ & $460 / 492$ \\
\hline & $1605 \mathrm{~cm}^{-1}$ & $1461 \mathrm{~cm}^{-1}$ & $1420 \mathrm{~cm}^{-1}$ & $1414 \mathrm{~cm}^{-1}$ \\
\hline \multirow{2}{*}{ Azul do nilo } & $625 / 657$ & $634 / 655$ & $637 / 660$ & $627 / 663$ \\
\hline & $779 \mathrm{~cm}^{-1}$ & $506 \mathrm{~cm}^{-1}$ & $547 \mathrm{~cm}^{-1}$ & $866 \mathrm{~cm}^{-1}$ \\
\hline \multirow[t]{2}{*}{ Cristal violeta } & $586 / 621$ & $588 / 621$ & $589 / 623$ & $590 / 626$ \\
\hline & $962 \mathrm{~cm}^{-1}$ & $904 \mathrm{~cm}^{-1}$ & $925 \mathrm{~cm}^{-1}$ & $975 \mathrm{~cm}^{-1}$ \\
\hline \multirow[t]{2}{*}{ Violeta de cresila } & $593 / 620$ & $599 / 621$ & $607 / 624$ & $611 / 624$ \\
\hline & $734 \mathrm{~cm}^{-1}$ & $591 \mathrm{~cm}^{-1}$ & $449 \mathrm{~cm}^{-1}$ & $341 \mathrm{~cm}^{-1}$ \\
\hline \multirow[t]{2}{*}{ Azul de toluidina } & $627 / 650$ & $629 / 646$ & $630 / 647$ & $631 / 650$ \\
\hline & $564 \mathrm{~cm}^{-1}$ & $418 \mathrm{~cm}^{-1}$ & $417 \mathrm{~cm}^{-1}$ & $463 \mathrm{~cm}^{-1}$ \\
\hline
\end{tabular}

A diminuição do deslocamento de Stokes com o aumento da cadeia carbônica do n-álcool utilizado indica uma menor solvatação do estado excitado dos corantes. Altos valores de deslocamento indicam uma reorganização da orientação dos grupos polares dos corantes. Há um aumento significativo para alguns dos corantes na intensidade de fluorescência quando se varia o solvente de metanol para propanol-1. Este efeito pode ser atribuído à diminuição da polaridade dos solventes, que aumenta a energia de ativação de conver- são do estado excitado planar para um estado de transferência de carga intramolecular aumentando o rendimento quântico de fluorescência. Um outro fato a ser considerado é que há um aumento da viscosidade o qual impede a rotação interna dos grupamentos presentes nas moléculas dos corantes, resultando num aumento da intensidade de fluorescência. Há ainda um outro aspecto a ser considerado nas variações espectrais observadas, sendo este a presença de ligações hidrogênio entre a molécula de corante e do solvente ${ }^{22-26}$. 


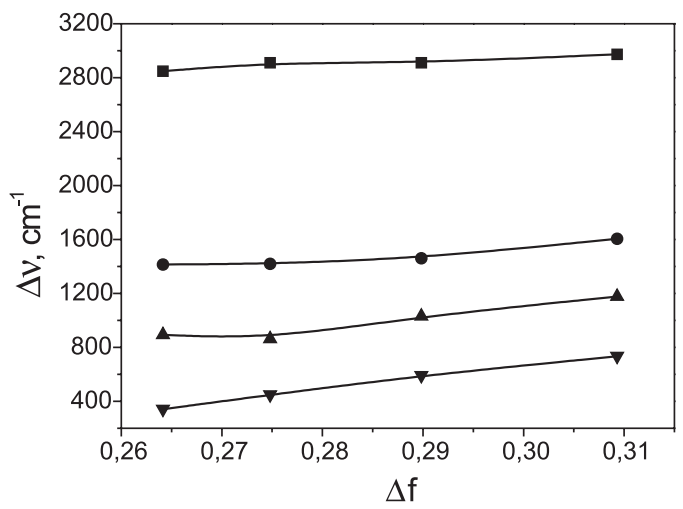

Figura 2. Gráfico de deslocamento de Stokes em função do parâmetro de polaridade do solvente para os corantes 9-aminoacridina (অ), proflavina (•), safranina $(\boldsymbol{\Delta})$ e cristal violeta $(\boldsymbol{\nabla}), T=298 \mathrm{~K}$

\section{Transferência de energia e cálculo de raio crítico de transferência de energia}

Os valores dos raios críticos de transferência $\left(\mathrm{R}_{0}\right)$ para os pares 9aminoacridina/safranina, 9-aminoacridina/azul do Nilo, 9-aminoacridina/ azul de toluidina, 9-aminoacridina/violeta de cresila, 9-aminoacridina/ cristal violeta, laranja de acridina/safranina, laranja de acridina/azul do Nilo, laranja de acridina/azul de toluidina, laranja de acridina/violeta de cresila, laranja de acridina/cristal violeta, proflavina/safranina, proflavina/ azul do Nilo, proflavina/azul de toluidina, proflavina/violeta de cresila e proflavina/cristal violeta, foram calculados com base nos espectros de absorção e emissão dos corantes.

Na obtenção dos valores de $\mathrm{R}_{0}$, utilizou-se os valores de rendimento quântico de fluorescência dos corantes (doadores) obtidos experimentalmente em metanol $(\mathrm{MeOH})$, etanol $(\mathrm{EtOH})$, propanol$1(\mathrm{PrOH})$ e butanol-1 $(\mathrm{BuOH})$, a partir dos padrões de fluorescência como o sulfato de quinina (região de emissão entre $400-600 \mathrm{~nm}$ ) e o violeta de cresila (região de emissão entre 600 - $800 \mathrm{~nm}$ ). Os valores obtidos são listados na Tabela 1. Os rendimentos quânticos foram calculados a partir da seguinte equação ${ }^{14,16,22}$ :

$\phi_{D}=\phi_{p} \frac{A^{D} \cdot A_{I f}^{p}}{A^{p} \cdot A_{I f}^{D}} \frac{n_{p}^{2}}{n_{D}^{2}}$

onde: $A_{I f}^{p}$ e $A_{I f}^{D}$ são as áreas integradas sob a curva do espectro de emissão corrigido do padrão e do doador, respectivamente e, $n_{p}^{2}$ e $n_{D}^{2}$ são, respectivamente, os quadrados dos índices de refração do solvente utilizados para o padrão e para o doador.

Os valores de $\mathrm{R}_{0}$ obtidos são apresentados na Tabela 4. Utilizouse a Equação 4 para se obter os valores de $\mathrm{R}_{0}$ para os diversos sistemas. O valor da eficiência do processo de transferência de energia é dado pela resolução das Equações 9 e 10, sendo que os valores de $\mathrm{C} / \mathrm{C}_{0}$ são as raízes desta. Os valores de $\mathrm{C} / \mathrm{C}_{0}$ foram obtidos no programa Maple V Release 5 (Waterloo Maple Inc.). O perfil típico da eficiência de transferência de energia é ilustrado na Figura 3 para um dos pares de corante estudado nos diversos solventes.

Utilizando a Equação 8 e os valores de $\mathrm{C}_{0}$, calculou-se os valores de $\mathrm{R}_{0}$ para os pares analisados, os quais são listados na Tabela 5. Os valores de $\mathrm{R}_{0}$ para os pares de corantes obtidos pela análise de decaimentos de fluorescência são apresentados na Tabela 6. As análises foram feitas a partir das Equações 5 e 6, e os resultados obtidos são a média das análises dos decaimentos para cada par doador/receptor em diferentes concentrações do receptor. Para cada medida foram feitos 5 decaimentos, sendo um na ausência de receptor e os
Tabela 4. Valores de $R_{0}(\AA)$ para os pares de corantes em diversos solventes, obtidos por sobreposição espectral, $\mathrm{T}=298 \mathrm{~K}$

\begin{tabular}{lrrrc}
\hline Solvente & $\mathrm{MeOH}$ & $\mathrm{EtOH}$ & $\mathrm{PrOH}$ & $\mathrm{BuOH}$ \\
\hline 9AA/VC & 81 & 96 & 105 & 61 \\
9AA/CV & 94 & 103 & 74 & 79 \\
9AA/AN & 51 & 94 & 91 & 73 \\
9AA/SAF & 109 & 96 & 98 & 67 \\
9AA/AT & 108 & 100 & 91 & 70 \\
\hline LA/VC & 58 & 67 & 63 & 70 \\
LA/CV & 103 & 99 & 71 & 74 \\
LA/AN & 85 & 100 & 74 & 67 \\
LA/SAF & 75 & 100 & 71 & 62 \\
LA/AT & 97 & 96 & 75 & 65 \\
\hline PROF/VC & 76 & 102 & 74 & 79 \\
PROF/CV & 91 & 98 & 79 & 81 \\
PROF/AN & 79 & 99 & 80 & 73 \\
PROF/SAF & 108 & 99 & 85 & 69 \\
PROF/AT & 105 & 94 & 91 & 73 \\
\hline
\end{tabular}

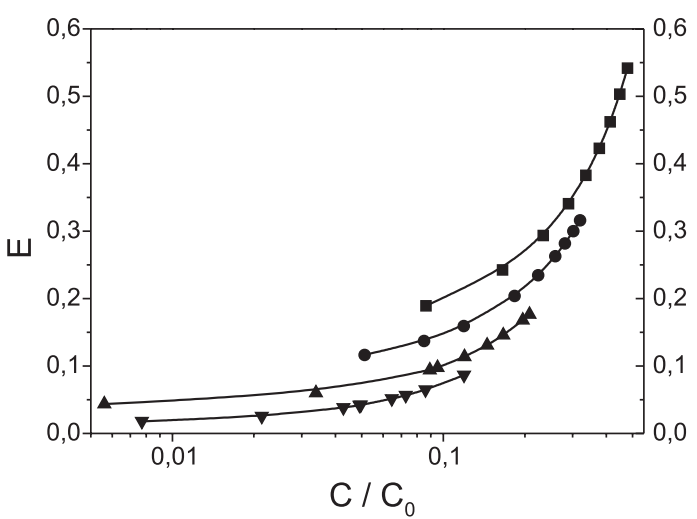

Figura 3. Perfil da eficiência do processo de supressão de fluorescência

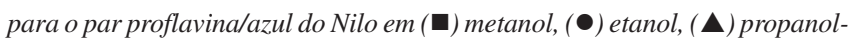
1 e $(\boldsymbol{\nabla})$ butanol-1, $T=298 \mathrm{~K}$

Tabela 5. Valores de $\mathrm{R}_{0}(\AA)$ para os pares de corantes em diversos solventes obtidos por fluorescência fotoestacionária, T = 298 K

\begin{tabular}{lllll}
\hline Solvente & $\mathrm{MeOH}$ & $\mathrm{EtOH}$ & $\mathrm{PrOH}$ & $\mathrm{BuOH}$ \\
\hline 9AA/VC & $74 \pm 1$ & $56 \pm 6$ & $75 \pm 7$ & $60 \pm 4$ \\
9AA/CV & $59 \pm 5$ & $57 \pm 4$ & $59 \pm 4$ & $57 \pm 2$ \\
9AA/AN & $62 \pm 5$ & $75 \pm 2$ & $71 \pm 8$ & $60 \pm 4$ \\
9AA/SAF & $75 \pm 3$ & $72 \pm 5$ & $74 \pm 6$ & $73 \pm 3$ \\
9AA/AT & $71 \pm 9$ & $75 \pm 4$ & $62 \pm 6$ & $68 \pm 6$ \\
\hline LA/VC & $76 \pm 2$ & $60 \pm 5$ & $65 \pm 3$ & $57 \pm 8$ \\
LA/CV & $72 \pm 5$ & $68 \pm 2$ & $73 \pm 5$ & $65 \pm 6$ \\
LA/AN & $59 \pm 7$ & $71 \pm 2$ & $70 \pm 9$ & $59 \pm 7$ \\
LA/SAF & $77 \pm 2$ & $78 \pm 9$ & $71 \pm 10$ & $69 \pm 9$ \\
LA/AT & $74 \pm 5$ & $66 \pm 2$ & $59 \pm 3$ & $66 \pm 7$ \\
\hline PROF/VC & $64 \pm 3$ & $60 \pm 2$ & $60 \pm 4$ & $64 \pm 3$ \\
PROF/CV & $65 \pm 3$ & $67 \pm 8$ & $67 \pm 10$ & $65 \pm 9$ \\
PROF/AN & $75 \pm 2$ & $65 \pm 3$ & $76 \pm 2$ & $77 \pm 5$ \\
PROF/SAF & $76 \pm 4$ & $75 \pm 7$ & $70 \pm 6$ & $71 \pm 4$ \\
PROF/AT & $65 \pm 5$ & $60 \pm 2$ & $74 \pm 1$ & $63 \pm 7$ \\
\hline
\end{tabular}


Tabela 6. Valores de $\mathrm{R}_{0}(\AA)$ para os pares de corantes em diversos solventes obtidos por fluorescência resolvida no tempo, $\mathrm{T}=298 \mathrm{~K}$

\begin{tabular}{lcccc}
\hline Solvente & $\mathrm{MeOH}$ & $\mathrm{EtOH}$ & $\mathrm{PrOH}$ & $\mathrm{BuOH}$ \\
\hline 9AA/VC & $69 \pm 4$ & $53 \pm 1$ & $69 \pm 3$ & $57 \pm 5$ \\
9AA/CV & $57 \pm 3$ & $54 \pm 2$ & $57 \pm 3$ & $56 \pm 4$ \\
9AA/NA & $62 \pm 2$ & $69 \pm 5$ & $64 \pm 3$ & $58 \pm 5$ \\
9AA/SAF & $69 \pm 3$ & $70 \pm 5$ & $73 \pm 4$ & $70 \pm 3$ \\
9AA/AT & $64 \pm 9$ & $68 \pm 5$ & $59 \pm 5$ & $63 \pm 5$ \\
\hline LA/VC & $71 \pm 4$ & $55 \pm 2$ & $56 \pm 2$ & $56 \pm 2$ \\
LA/CV & $64 \pm 3$ & $63 \pm 4$ & $68 \pm 4$ & $61 \pm 2$ \\
LA/NA & $59 \pm 3$ & $61 \pm 2$ & $67 \pm 3$ & $58 \pm 5$ \\
LA/SAF & $71 \pm 2$ & $73 \pm 3$ & $71 \pm 3$ & $69 \pm 3$ \\
LA/AT & $74 \pm 5$ & $62 \pm 2$ & $58 \pm 2$ & $64 \pm 4$ \\
\hline PROF/VC & $60 \pm 5$ & $54 \pm 1$ & $54 \pm 7$ & $56 \pm 3$ \\
PROF/CV & $62 \pm 2$ & $65 \pm 1$ & $65 \pm 4$ & $64 \pm 4$ \\
PROF/AN & $73 \pm 2$ & $65 \pm 3$ & $72 \pm 2$ & $72 \pm 3$ \\
PROF/SAF & $74 \pm 3$ & $71 \pm 3$ & $68 \pm 4$ & $70 \pm 2$ \\
PROF/AT & $61 \pm 2$ & $58 \pm 1$ & $66 \pm 1$ & $62 \pm 2$ \\
\hline
\end{tabular}

outros 4 na presença de receptor em concentrações crescentes deste. As análises dos decaimentos com transferência de energia foram efetuadas utilizando como parâmetro pré-determinado o tempo de vida do doador na ausência de receptor. Neste caso, pode-se verificar a influência da contribuição de difusão dos corantes no processo de transferência de energia ${ }^{27-29}$, a qual é mais importante para sistemas com tempo de vida de fluorescência longo. Quando a taxa de decaimento exponencial em tempos longos se iguala ao inverso do tempo de vida do corante doador na ausência de supressor, o processo de supressão por difusão pode ser considerado mínimo, não sendo então necessário corrigir os valores de $\mathrm{R}_{0}$ em função do efeito de difusão. Um exemplo de decaimento na ausência e presença de supressor pode ser visto na Figura 4, para o par 9AA/VC em metanol.

Com o objetivo de comparar os resultados de $\mathrm{R}_{0}$ obtidos pelos diferentes métodos, estudou-se a correlação entre os valores de raio crítico de transferência de energia $\left(\mathrm{R}_{0}\right)$. A Figura 5 mostra um gráfico de correlação entre os valores obtidos via fluorescência estacionária e resolvida no tempo. A partir da análise da correlação, conclui-se que as melhores técnicas de determinação de $\mathrm{R}_{0}$ são a de fluorescência estacionária (via supressão) e fluorescência resolvida no tempo (também via supressão), dado que os valores encontrados nestes dois métodos possuem boa concordância entre si. A análise dos dados apresenta um valor de coeficiente de correlação de 0,92 com um erro em relação à média de $2,5 \AA$ A . Os valores de $\mathrm{R}_{0}$ estacionário diferem em relação aos valores de $\mathrm{R}_{0}$ resolvido no tempo, sendo maiores por um fator de $5 \pm 3 \AA$ em média. Os dados de $\mathrm{R}_{0}$ obtidos por sobreposição espectral possuem pouca correlação com os dados obtidos pelas outras duas técnicas. Este fato provavelmente é devido a erros decorrentes durante a obtenção dos dados espectrais e dos valores de rendimento quântico de fluorescência.

\section{CONCLUSÕES}

Neste trabalho, o raio crítico de Förster $\left(\mathrm{R}_{0}\right)$ para uma série de pares de corantes catiônicos foi calculado por três processos diferentes. Os resultados mostraram a existência de uma melhor concordância de valores de $\mathrm{R}_{0}$ obtidos pelas técnicas de fluorescência estacionária e resolvida no tempo. Desta forma, os métodos de avaliação de $\mathrm{R}_{0}$ baseados em fluorescência estacionária e resolvida no tempo são os mais indicados para o cálculo deste parâmetro vinculado ao processo de transferência de energia eletrônica.
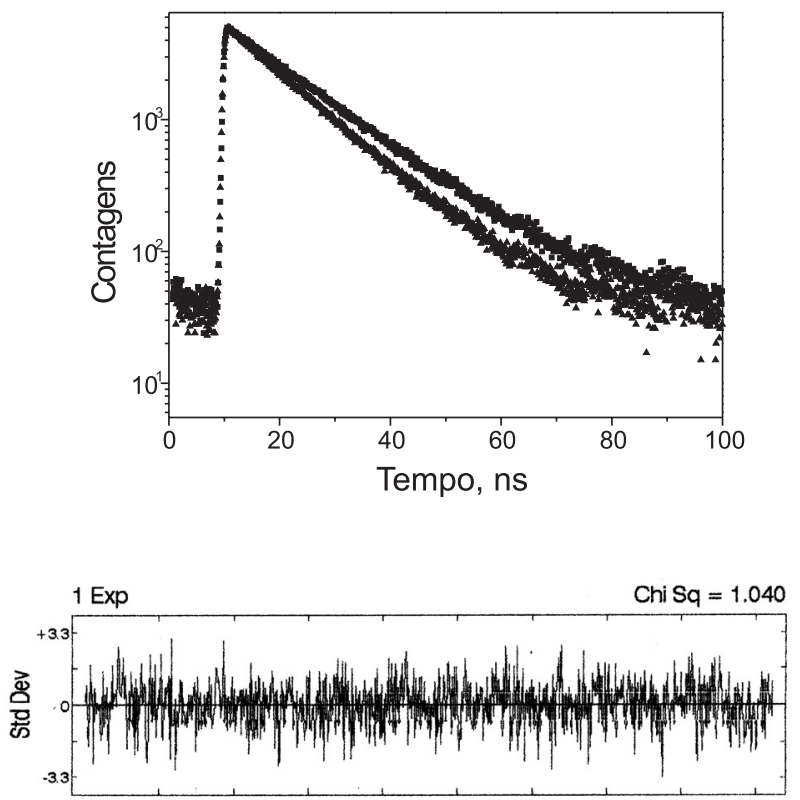

(a)

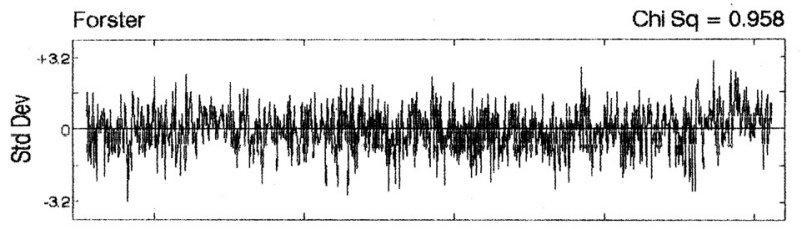

(b)

Figura 4. Decaimento e ajuste para a 9-aminoacridina/violeta de cresila em metanol, (a) ausência de violeta de cresila, $\tau_{0}=13,89 \mathrm{~ns}$ e (b) 8,33.10 $0^{-4} \mathrm{M}$ de violeta de cresila, $\Gamma=0,286, T=298 \mathrm{~K}$

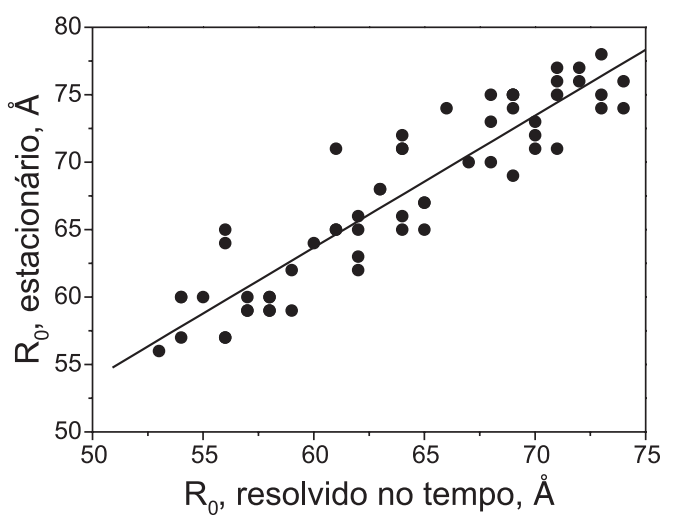

Figura 5. Gráfico de correlação entre os valores de raio crítico de transferência de energia $\left(R_{0}\right)$, obtidos via fluorescência estacionária e resolvida no tempo, $T=298 \mathrm{~K}$

\section{AGRADECIMENTOS}

H. P. M. Oliveira e A. Machulek Jr. agradecem à FAPESP pelas bolsas de doutorado e mestrado recebidas. A. O. Legendre agradece ao $\mathrm{CNPq}$ pela bolsa de iniciação científica recebida.

\section{REFERÊNCIAS}

1. Berkovic, G.; J. Chem. Educ. 1984, 61, 832. 
2. Niu, E. P.; Ghiggino, K. P.; Smith, T. A.; Mau, A. W. H.; J. Lumin. 1990, $46,191$.

3. Koglin, P. K. F.; Miller, D. J.; Stelnwandel, J.; Hauser, M.; J. Phys. Chem. 1981, 85, 2363

4. Gehlen, M. H.; Buchviser, S. F.; Rodrigues, M. R.; Neumann, M. G.; Quim. Nova 1998, 21, 794.

5. Drake, J. M.; Klafter, J.; Levitz, P.; Science 1991, 251, 1574.

6. Hhadjianestis, J.; Nikokavouras, J.; J. Photochem. Photobiol., A 1993, 69, 337.

7. Sánchez, F. G.; Ruiz, C. C.; J. Lumin. 1996, 69, 179.

8. Marcus, A. H.; Fayer, M. D.; J. Chem. Phys. 1991, 94, 5622.

9. Berlman, I. B.; Handbook of Fluorescence Spectra of Aromatic Molecules, $2^{\text {nd }}$ ed, Academic Press: New York, 1971.

10. Barzykin, A. V.; Tachiya, M.; J. Chem. Phys. 1995, 102, 3146.

11. Neumann, M. G.; Pastre, I. A.; Sol. Energy Mater. Sol. Cells 1987, 36, 431.

12. Berberan-Santos, M. N.; Prieto, M. J. E.; J. Chem. Soc., Faraday Trans. 2 1987, 83, 1391.

13. Gehlen, M. H.; De Schryver, F. C.; Chem. Rev. 1993, 93, 199.

14. Lakowicz, J. R.; Principles of Fluorescence Spectroscopy, $2^{\text {nd }}$ ed, Kluwer Academic/Plenum Publishers: New York, 1999.

15. Förster, T.; Discuss. Faraday Soc. 1959, 27, 7.
16. Scaiano, J. C.; Handbook of Organic Photochemistry; CRC Press: Boca Raton, 1989, vol. 1 e 2.

17. Nagasawa, Y.; Yoshihara, K.; J. Chem. Phys. 1994, 101, 5717.

18. Douhal, A.; J. Phys. Chem. 1994, 98, 13131.

19. Yoshihara, K.; Nagasawa, Y.; Yartsev, A.; Kumazaki, S.; Kandori, H.; Johnson, A. E.; Tominaga, K.; J. Photochem. Photobiol., A 1994, 80, 169.

20. Grofcsik, A.; Kubinyi, M.; Jones, W. J.; Chem. Phys. Lett. 1996, 250, 261.

21. Dutta, A. K.; Kamada, K.; Ohta, K.; J. Photochem. Photobiol., A 1996, 93, 57.

22. Yekta, A.; Duhamel, J.; Brochard, P.; Adiwidjaja, H.; Winnik, M.; Macromolecules 1993, 26, 1829.

23. Neumann, M. G.; Tierra, M. J.; Quim. Nova 1993, 16, 280.

24. Grofcsik, A.; Kubinyi, M.; Jones, W. J.; J. Chem. Soc., Faraday Trans. 1992, 88, 1101.

25. Nakashima, K.; Fujimoto, Y.; Photochem. Photobiol. 1994, 60, 563.

26. Mialocq, J. C.; Hébert, P.; Armand, X.; Bonneau, R.; Morand, J. P.; J. Photochem. Photobiol., A 1991, 56, 323.

27. Gösele, U.; Hauser, M.; Klein, U. K. A.; Frey, R.; Chem. Phys. Lett. 1975, 34,519 .

28. Gösele, U.; Chem. Phys. Lett. 1976, 43, 61 .

29. Klein, U. K. A.; Frey, R.; Hauser, M.; Gösele, U.; Chem. Phys. Lett. 1976, $41,139$. 